\title{
The melanocortin receptors and their accessory proteins
}

\author{
Shwetha Ramachandrappa, Rebecca J. Gorrigan, Adrian J. L. Clark and Li F. Chan* \\ Centre for Endocrinology, William Harvey Research Institute, Queen Mary University of London, Barts and The London School of Medicine and Dentistry, \\ London, UK
}

\section{Edited by:}

Jae Young Seong, Korea University,

South Korea

\section{Reviewed by:}

Akiyoshi Takahashi, Kitasato

University, Japan

Robert Dores, University of

Minnesota, USA

\section{*Correspondence:}

Li F. Chan, Centre for Endocrinology,

William Harvey Research Institute,

Queen Mary University of London,

Barts and The London School of

Medicine and Dentistry, Charterhouse

Square, London EC1M 6BQ, UK

e-mail: I.chan@qmul.ac.uk
The five melanocortin receptors (MCRs) named MC1R-MC5R have diverse physiological roles encompassing pigmentation, steroidogenesis, energy homeostasis and feeding behavior as well as exocrine function. Since their identification almost 20 years ago much has been learnt about these receptors. As well as interacting with their endogenous ligands the melanocortin peptides, there is now a growing list of important peptides that can modulate the way these receptors signal, acting as agonists, antagonists, and inverse agonists. The discovery of melanocortin 2 receptor accessory proteins as a novel accessory factor to the MCRs provides further insight into the regulation of these important $\mathrm{G}$ protein-coupled receptor.

Keywords: melanocortin receptors, accessory proteins, adrenal cortex, familial glucocorticoid deficiency, obesity

\section{THE MELANOCORTIN SYSTEM}

The melanocortin receptors (MCRs) are a family of five G proteincoupled receptors (GPCRs; MC1R-MC5R) expressed in diverse tissues, which serve discrete physiological functions. Early studies delineated a preliminary model where the receptors were activated by melanocortin peptides, derived from post-translational processing and proteolytic cleavage of the precursor protein proopiomelanocortin (Eipper and Mains, 1980). Receptor activation increased intracellular cyclic adenosine monophosphate (cAMP) levels triggering several downstream signaling pathways. Subsequent research into melanocortin signaling has added several additional tiers of complexity to this basic schema. It has emerged that many of the MCRs bind to endogenous peptides beyond the melanocortin peptides, which can act as agonists, antagonists, partial agonists, and even inverse agonists at these receptors (Cone, 2006). Furthermore, although the cAMP pathway continues to serve as the predominant readout of MCR function in many studies, numerous other intracellular signaling cascades for example the mitogen-activated protein kinase pathway have also been implicated in melanocortin signaling (Vongs et al., 2004; Sutton et al., 2005; Patten etal., 2007; Rodrigues et al., 2009). An additional feature of MCR signaling which has come to light over recent years is the presence of accessory proteins which regulate receptor function. These observations have broadened our understanding of MCR signaling, unveiling a highly specialized system, which on a cellular level enables individual cells to generate a complex co-ordinated response to the unique complement of ligands in their microenvironment (Table 1).

\section{MC1R}

MC1R is expressed in the melanocytes of the skin and hair follicles. Activation of MC1R results in a switch from red/yellow pheomelanin pigment production to the production of brown/black eumelanin pigment; it also promotes cell proliferation, DNA repair, and cell survival. Variants in MC1R are associated with red hair color, fair skin, and increased skin cancer risk (Valverde et al., 1995). Many of these variant receptors have been studied in vitro and their function, as measured by cAMP production in response to [Nle4,D-Phe7]- $\alpha$-MSH (NDP-MSH), has been shown to be impaired compared to wild-type. Until recently MC1R was thought to be exclusively activated by alpha-MSH endogenously produced by keratinocytes in response to ultraviolet radiation. Human $\beta$-defensin 3 has since emerged as a novel endogenous MC1R ligand whose binding appears to initiate a discrete complement of intracellular signaling pathways in vitro (Beaumont et al., 2012; Swope et al., 2012). The physiological importance of this interaction remains to be seen. MC1R is also expressed in macrophages and in adipocytes where its role is less clearly defined. At extremely high plasma adrenocorticotropin hormone (ACTH) concentrations, ACTH activation of MC1R leads to hyperpigmentation observed in patients with familial glucocorticoid deficiency (FGD) (Turan et al., 2012). The study of the genetic determinants of coat color in animal models led to the discovery of agouti (also known as agouti signaling peptide), a high-affinity antagonist of the MC1R (Lu et al., 1994). The identification of an endogenous, physiologically relevant GPCR antagonist marked the beginning of a paradigm shift in our understanding of GPCR signaling. Later work demonstrated the complexity of this molecule by exploring its ability to act as an inverse agonist (Vage et al., 1997), inhibiting constitutively active MC1R.

\section{MC2R}

$\mathrm{MC} 2 \mathrm{R}$ is predominantly expressed in the adrenal gland where it promotes the expression of steroidogenic enzymes in response to binding plasma ACTH. Mutations in MC2R result in FGD (Clark etal., 1993; Tsigos etal., 1993). This is a rare, lifethreatening autosomal recessive disorder of adrenal resistance to ACTH wherein affected individuals have low serum levels of cortisol despite the presence of extremely high circulating levels of plasma ACTH. Patients typically present within a few months 
Table 1 |The members of the melanocortin receptor family, expression, action, and phenotype of individuals with mutations.

\begin{tabular}{|c|c|c|c|c|}
\hline Receptor & Main sites of expression & Physiological functions & $\begin{array}{l}\text { Disease phenotype of patients with } \\
\text { loss-of-function mutations }\end{array}$ & OMIM \\
\hline MC1R & Melanocytes, macrophage & Pigmentation, inflammation & Increased risk of skin cancers & 155555 \\
\hline MC2R & Adrenal cortex & Adrenal steroidogenesis & Familial glucocorticoid deficiency & 202200 \\
\hline MC3R & $\begin{array}{l}\text { Central nervous system (CNS), } \\
\text { gastrointestinal (GI) tract, Kidney }\end{array}$ & Energy homeostasis, inflammation & Obesity & 155540 \\
\hline MC4R & CNS, spinal cord & $\begin{array}{l}\text { Energy homeostasis, appetite } \\
\text { regulation, erectile function }\end{array}$ & Obesity & 155541 \\
\hline MC5R & Lymphocytes, exocrine cells & $\begin{array}{l}\text { Exocrine function, regulation of } \\
\text { sebaceous glands }\end{array}$ & $\begin{array}{l}\text { Decreased production of sebaceous lipids } \\
\text { in mice }\end{array}$ & 600042 \\
\hline
\end{tabular}

of life with symptoms of cortisol deficiency including recurrent infections, hypoglycemia, convulsions, failure to thrive, and shock. Milder forms of the disease have been observed to present later in life (Metherell et al., 2009; Hughes et al., 2010; Meimaridou et al., 2012). The classical presentation of FGD comprises isolated perturbation of the glucocorticoid axis; however, a small number of cases have been described where this is accompanied by a disorder of mineralocorticoid secretion (Lin et al., 2007; Chan et al., 2009a).

Mutations in MC2R account for 25\% of cases of FGD. The remaining $75 \%$ of patients with FGD in whom mutations in MC2R have been excluded have formed the subject of genetic approaches to elucidate other causative loci. To date, mutations in three further genes have been associated with FGD. Mutations in the melanocortin 2 receptor accessory protein (MRAP), a single pass transmembrane protein implicated in MC2R function account for approximately 15-20\% of cases of FGD (Metherell et al., 2005). Mutations in steroidogenic acute regulatory (STAR) protein are known to give rise to lipoid congenital adrenal hyperplasia, a severe form of adrenal insufficiency characterized by both glucocorticoid and mineralocorticoid deficiency together with gonadal deficiency; however, STAR mutations have also been identified in a number of patients with FGD suggesting that mutations in this gene can give rise to a spectrum of clinical phenotypes encompassing FGD (Metherell et al., 2009). Recently, mutations in nicotinamide nucleotide transhydrogenase (NNT) a mitochondrial membrane constituent which is involved in detoxification of reactive oxygen species were also associated with FGD (Meimaridou et al., 2012). Notably, mutations in minichromosome maintenance-deficient 4 (MCM4) which forms part of a protein complex which is essential for DNA replication and genome stability are associated with a variant of FGD found in the Irish Traveller population where adrenal failure is accompanied by short stature, chromosome instability, and natural killer cell dysfunction (O'Riordan et al., 2008; Gineau et al., 2012; Hughes et al., 2012).

\section{MC3R}

MC3R is primarily expressed in the central nervous system where it is found in the hypothalamus and the limbic regions (RoselliRehfuss et al., 1993). Targeted deletion of MC3R in murine models results in animals with increased fat mass, reduced lean mass, and reduced physical activity in the absence of hyperphagia (Butler et al., 2000; Chen et al., 2000). Additionally, these animals exhibit accelerated weight gain when placed on a high fat diet which is independent of hyperphagia implying that MC3R may be involved in nutrient partitioning. An emerging aspect of the MC3R deficient phenotype is that when these animals are subjected to food restriction regimes they exhibit impaired synchronized oscillation of the transcription factors which regulate liver clock activity and metabolism, utilize carbohydrates inappropriately, and fail to demonstrate the food anticipatory behaviors which are seen in wild-type mice (Sutton et al., 2008, 2010). These observations have led to the suggestion that MC3R is involved in coordinating appropriate homeostatic metabolic and behavioral responses to nutrient cues. Interestingly, despite focused investigation, rare variants at the MC3R locus have not been definitively associated with monogenic obesity in humans (Seng Lee et al., 2007; Mencarelli et al., 2011).

\section{MC4R}

MC4R is widely expressed in the central nervous system where it is abundant in several regions including the paraventricular nucleus (PVN) of the hypothalamus (Mountjoy et al., 1994). Targeted deletion of MC4R in a mouse model results in animals which are severely obese, with hyperphagia and reduced energy expenditure (Huszar et al., 1997). In an informative study, Cre-lox technology was used to selectively re-express MC4R at endogenous levels in the PVN and the amygdale of MC4R knockout animals (Balthasar et al., 2005). This was able to partially rescue their obese phenotype by normalizing their food intake suggesting that MC4Rs in these regions are responsible for regulating eating behavior while MC4Rs in anatomically distinct neuronal populations are relevant to its control of energy expenditure.

The PVN is a key integration center for diverse signals which impact upon energy balance and is densely innervated by the arcuate nucleus of the hypothalamus. The release of proopiomelanocortin (POMC)-derived peptides from neurons in the arcuate nucleus, which are stimulated by leptin results in the activation of MC4R signaling in the PVN (Cheung etal., 1997; Schwartz et al., 1997). Uniquely for a biological system, a physiologically relevant endogenous antagonist and inverse agonist of MC4R (as well as MC3R) signaling is also produced in the hypothalamus, 
agouti-related peptide (AGRP; Nijenhuis et al., 2001; Breit et al., 2006). ARGP is produced by leptin responsive neurons in the arcuate nucleus that are inhibited by leptin. The presence of endogenous agonists and antagonists for this receptor enables MC4R signaling in the PVN to be delicately balanced.

Mutations in the MC4R are the most prevalent form of monogenic obesity identified to date accounting for up to $6 \%$ of patients with severe obesity (Vaisse et al., 1998; Yeo et al., 1998). The signaling capacities of specific mutant receptors studied in vitro correlates with the severity of the phenotype of corresponding MC4R deficient individuals (Farooqi et al., 2003). This demonstrates that the system is sensitive to degrees of loss in function at the level of the receptor. In addition to rare variants which cause highly penetrant forms of monogenic obesity, common variants in MC4R have been associated with body mass index (BMI) in genome wide association scans suggesting that variation at this locus also contributes to obesity in the general population (Willer et al., 2009).

\section{MC5R}

The MC5R is widely expressed in peripheral tissues. Mice lacking MC5R are unable to produce the full complement of sebaceous lipids which constitute the water repellent component of their coats (Chen et al., 1997). As a result they demonstrate impaired water repulsion and thermoregulation. Studies in these mice have also suggested that MC5Rs play a role in the generation of pheromones which in turn influences aspects of behavior (Morgan et al., 2004; Morgan and Cone, 2006). High levels of MC5R are found in multiple exocrine tissues where they are thought to regulate the synthesis and secretion of a diverse range of exocrine products.

\section{THE MELANOCORTIN RECEPTOR ACCESSORY PROTEINS THE DISCOVERY OF MRAP}

The functional properties of the MCRs have been extensively examined in in vitro expression systems. Comparable studies of MC2R function were initially impeded by difficulties in expressing functional receptor in conventionally used cell lines. It became apparent that unlike other MCRs functional MC2R could only be expressed in cell lines of adrenal origin, suggesting that its expression was contingent upon the presence of a tissue-specific protein. This factor was identified in a study of genetic loci associated with FGD. Mutations in MC2R were known to lead to this inherited syndrome of ACTH resistance, but were only able to account for approximately $25 \%$ of cases. Whole genome single nucleotide polymorphism (SNP) analysis in two affected consanguineous families in whom mutations in MC2R had been excluded identified a region of interest encompassing a novel protein, which was highly expressed in the adrenal gland. In vitro studies confirmed that expression of this protein alongside MC2R enabled functional MC2R receptor expression in heterologous cell types and it was subsequently named MRAP (Metherell et al., 2005). Fifteen to twenty percent of cases of FGD are now known to be caused by mutations in MRAP.

\section{MRAP STRUCTURE}

Human MRAP is a single pass transmembrane protein which exists as two isoforms which differ in their C-termini. Both isoforms have been shown by reverse transcription polymerase chain reaction (RT-PCR) in a cDNA panel derived from human tissues to be expressed at high levels in the adrenal gland (Metherell et al., 2005). MRAP expression has also been demonstrated in a variety of other human tissues including testis, breast, thyroid, lymph nodes, ovary, and skin (Metherell et al., 2005). Immunofluorescence microscopy staining of cultured cells which endogenously express MRAP, using antibodies raised against $\mathrm{N}$-terminal and C-terminal MRAP peptides, have shown that both regions of MRAP are present at the cell surface (Sebag and Hinkle, 2007). In vitro studies where differentially epitope-tagged forms of the protein were co-expressed in cells and their interaction and orientation were studied show that MRAP monomers form unique anti-parallel homodimeric structures (Sebag and Hinkle, 2007; Cooray et al., 2008). These dimers can be visualized in the endoplasmic reticulum and at the plasma membrane in live cells using biomolecular fluorescence complementation techniques (Sebag and Hinkle, 2009b, 2010). The deletion of amino acids 31-37 in mouse MRAP which directly precede the transmembrane portion of the protein abolishes its ability to homodimerize suggesting that this region of the protein constitutes a critical component of the interface between the two monomers (Sebag and Hinkle, 2009b). This mutant is unable to assist trafficking of the MC2R to the cell surface, suggesting that this unique conformation is essential to certain aspects of MRAP function (Figure 1).

\section{MRAP AND MC2R}

Cell lines derived from adrenocortical tissue express both MC2R and MRAP and generate cAMP in response to treatment with ACTH. This response is abrogated by MRAP knockdown in these cells (Cooray et al., 2008). MRAP expression is necessary and sufficient for the functional expression of MC2R constructs in heterologous cell lines such as chinese hamster ovary $(\mathrm{CHO})$ cells and SK-N-SH cells which are derived from ovarian and neuroblastoma tissue, respectively and do not endogenously express measurable levels of MRAP (Metherell et al., 2005; Sebag and Hinkle, 2007).

\section{MRAP FACILITATES TRAFFICKING OF THE MC2R TO THE CELL SURFACE}

When epitope-tagged MC2R is expressed in $\mathrm{CHO}$ cells it is visualized in the endoplasmic reticulum, however, when it is expressed alongside MRAP it is predominantly localized to the plasma membrane (Metherell et al., 2005; Sebag and Hinkle, 2007; Webb et al., 2009). Consistent with this finding, in cells of adrenal origin with endogenously expression of MRAP, MC2R can be demonstrated at the cell surface. Western blotting of cell lysates yields a different complement of MC2R species in the presence and absence of MRAP, suggesting that MRAP may enhance receptor trafficking to the cell surface by promoting post-translational modification (Sebag and Hinkle, 2007). Deletion mutants of the N-terminal region of human MRAP which lack amino acids 1-24 or 1-35 are unable to promote MC2R trafficking to the cell surface suggesting that the $\mathrm{N}$-terminal region plays a prominent role in receptor trafficking (Webb et al., 2009).

\section{MRAP FACILITATES MC2R SIGNALING}

In vitro experiments examining MC2R signaling in the presence of various MRAP mutants suggest that the ability of MRAP to 


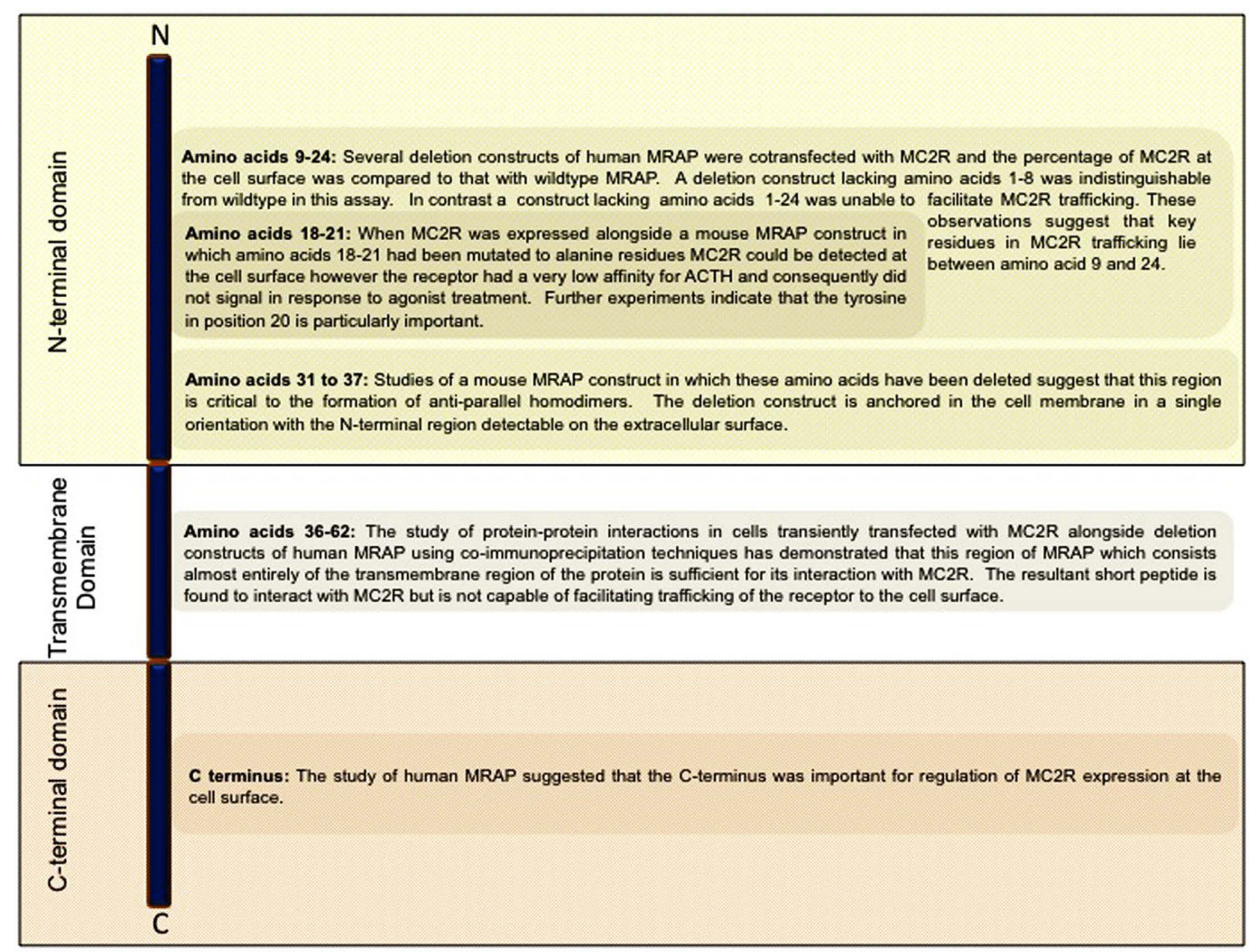

FIGURE 1 | Schematic representation of the domain structure of MRAP highlighting regions of functional significance in MRAP dimerization and in its interaction with MC2R (Sebag and Hinkle, 2009b; Webb et al., 2009).

promote MC2R trafficking to the cell surface does not wholly account for its role in facilitating MC2R signaling. Amino acids within the N-terminal region of MRAP appear to influence MC2R signaling independently of enhancing receptor trafficking. Specifically, MC2R receptor is able to traffic to the cell surface but is unable to bind to ACTH in the presence of a deletion mutant of mouse MRAP in which amino acids 18-21 have been mutated to alanine residues (Sebag and Hinkle, 2009b).

\section{MRAP AND MC2R COMPLEXES}

Protein complexes containing both MRAP and MC2R can be reciprocally isolated using co-immunoprecipitation techniques in transiently transfected cells (Metherell et al., 2005; Webb et al., 2009). Analogous experiments using MRAP deletion mutations indicate that the transmembrane region of MRAP interacts with MC2R (Webb et al., 2009). A model has emerged wherein MRAPMC2R complexes interact with one another to form higher order complexes which facilitate MC2R function. BRET techniques have been used to explore some of the interactions within these complexes in more detail in live cultured cells with specific reference to the effect of the MC2R agonist ACTH. These experiments have shown that ACTH enhances the interaction between MC2R homodimers and MRAP-MC2R heterodimers (Cooray et al., 2011). Schematic representation of the role of MRAP in MC2R signaling is shown in Figure 2.

\section{MRAP INTERACTIONS WITH OTHER MEMBERS OF THE MELANOCORTIN RECEPTOR FAMILY}

MRAP can be demonstrated to form protein complexes with all five members of the MCR family using co-immunoprecipitation techniques in cultured cells (Chan etal., 2009b). Aside from $M C 2 R$, the remaining members of the MCR family are capable of efficiently trafficking to the cell surface when expressed in $\mathrm{CHO}$ cells. In a series of experiments using human constructs, co-expression of MRAP alongside either MC1R or MC3R in CHO cells was not found to alter cell surface expression of the receptors or influence their ability to generate cAMP in response to agonist treatment. In contrast, the cell surface expression and signaling capacities of both MC4R and MC5R were found to be significantly reduced in the presence of MRAP in these cells (Table 2). Signaling in these experiments was assessed by measuring cAMP generation using a competitive protein binding assay in response to treatment with a single concentration of agonist (Chan et al., 2009b). When the signaling properties of MC4R in the presence of MRAP were studied by measuring cAMP generation with a luciferase assay across a range of agonist concentrations using mouse constructs, co-expression of MRAP was not found to influence MC4R signaling (Sebag and Hinkle, 2010). Further examination of this interaction is needed to definitively understand whether MRAP interacts with MC4R in vivo and to determine the impact of this interaction on MC4R signaling. The in vitro interaction of 


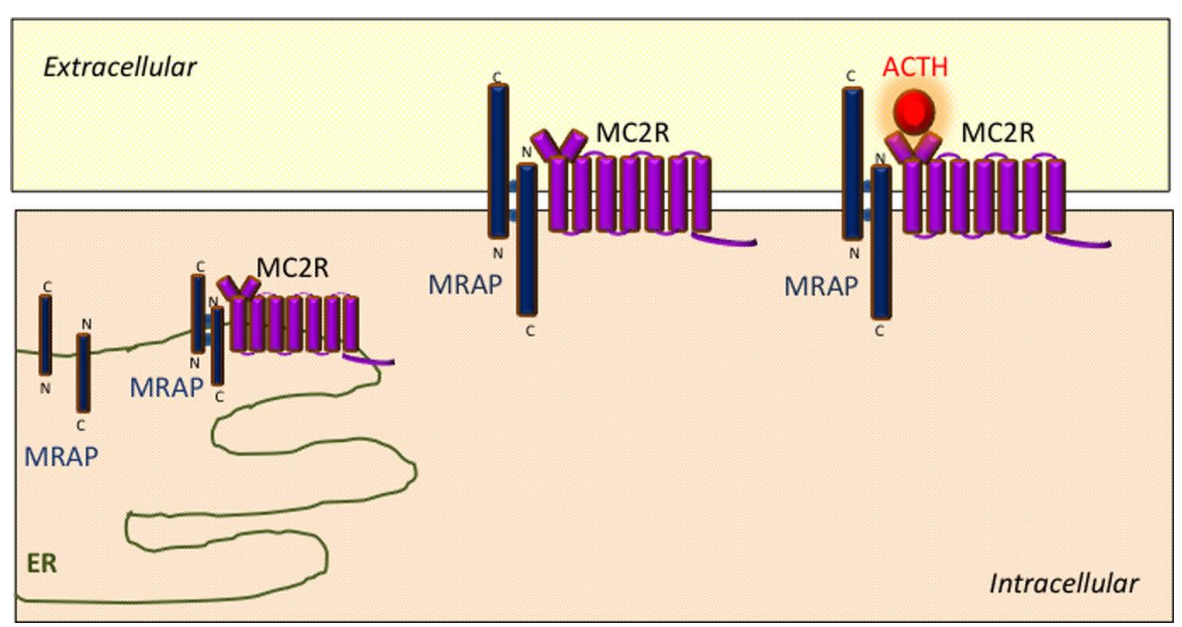

FIGURE 2 | Schematic representation of the role of MRAP in MC2R signaling. MRAP monomers form unique anti-parallel homodimeric complexes in the endoplasmic reticulum which interact with $M C 2 R$ and assist its trafficking to the cell surface. The MRAP/MC2R structure is capable of binding ACTH at the cell surface and initiating intracellular signaling.

MRAP and MC5R has been explored more fully using bimolecular fluorescence complementation techniques (Sebag and Hinkle, 2009a). When MC5R constructs are expressed in CHO cells MC5R monomers interact to from homodimers or oligomers. When MRAP is expressed alongside MC5R fewer multimeric complexes are formed and fewer receptors are expressed at the cell surface. This data suggests that MRAP is capable of interacting with members of the MCR family beyond MC2R and may differentially regulate aspects of their function. However, patients with MRAP mutations present with isolated glucocorticoid deficiency apparently without any other additional symptoms (Chung et al., 2010). One patient with FGD caused by a mutation in MRAP was described as being concomitantly obese, although the significance of this is unclear as the rest of the family had similarly high BMIs (Rumie et al., 2007).

\section{MRAP IN ADIPOCYTES}

Prior to its advent as an accessory factor for MC2R in the adrenal gland, MRAP was identified as a novel protein expressed in mouse adipocytes (Xu et al., 2002). Both isoforms of MRAP have subsequently been shown by RT-PCR to be expressed in human adipocyte tissue (Metherell et al., 2005). The function of MRAP in these cells is not known. Given its established role in facilitating
MC2R function in the adrenal gland it is likely that its function is related to the melanocortin system. The distribution of epitopetagged MRAP within cultured adipocytes is responsive to insulin treatment suggesting that its role is contingent upon metabolic cues. Notably, gross metabolic dysfunctions have not been reported in patients with mutations in MRAP; however, this may reflect functional redundancy with the related protein MRAP2 in this tissue whose expression in adipocytes has not yet been investigated. The components of the melanocortin system which are expressed in adipocyte tissue show species-specific differences. 3T3L1 cells which are cells of murine origin that are commonly used in the study of adipocyte function express both MC2R and MC5R. Human adipose tissue samples have been shown to express MC1R and low levels of MC4R and MC5R (Hoch et al., 2007). Treatment of 3T3L1 cells with melanocortin ligands promotes lipolysis and cytokine production in vitro, however, this observation has not been replicated in human mesenchymal cell derived adipocytes (Hoch et al., 2008; Jun et al., 2010; Moller et al., 2011).

\section{IDENTIFICATION OF MRAP2}

Following the discovery of MRAP a related gene of unknown function with a comparable structure and a high degree of $\mathrm{N}$-terminal and C-terminal sequence similarity was identified on

Table 2 | In vitro effects of MRAP on melanocortin receptors, based on data derived from Metherell et al. (2005) Sebag and Hinkle (2007, 2009a, 2010), Chan et al. (2009b) and Cooray et al. (2011).

\begin{tabular}{|c|c|c|}
\hline Receptor & Effect of MRAP on trafficking & Effect of MRAP on signaling (cAMP generation) \\
\hline MC1R & No change in cell surface expression & No effect in response to NDP-MSH \\
\hline $\mathrm{MC} 2 \mathrm{R}$ & Required for surface expression and receptor maturation & Required of receptor signaling in response to $\mathrm{ACTH}$ \\
\hline MC3R & No change in cell surface expression & No effect in response to NDP-MSH \\
\hline MC4R & Reduction in the surface expression & $\begin{array}{l}\text { Reduction in response to NDP-MSH } \\
\text { (No change was seen in another study) }\end{array}$ \\
\hline MC5R & Reduction in the surface expression & Reduction in response to NDP-MSH \\
\hline
\end{tabular}


chromosome 6 and was named MRAP2 (Metherell et al., 2005; Chan et al., 2009b). MRAP2 is a single pass transmembrane protein, in humans it has been shown by RT-PCR to be expressed in the brain and the adrenal gland (Chan et al., 2009b). Western blotting of mouse tissues resolves a single band of MRAP2 reactive species whose molecular weight corresponds to a dimer suggesting that endogenous MRAP2 exists in dimeric complexes (Chan et al., 2009b). MRAP2 undergoes N-linked glycosylation at its N-terminus and is thought to form anti-parallel homodimeric complexes analogous to those formed by MRAP (Chan et al., 2009b; Sebag and Hinkle, 2009a, 2010). MRAP2 constructs can be visualized in the endoplasmic reticulum and the plasma membrane of transiently transfected $\mathrm{CHO}$ cells (Chan et al., 2009b).

\section{MRAP2 AND MC2R SIGNALING}

The physiological role of MRAP2 is not yet known. In view of the structural similarities between MRAP and MRAP2 and its concurrent expression in the adrenal gland, the interaction between MRAP2 and MC2R has been explored in detail. As discussed previously, when MC2R constructs are expressed in $\mathrm{CHO}$ cells in isolation the receptors are unable to reach the cell surface. Concurrent expression of MRAP facilitates MC2R trafficking to the cell surface. At the cell surface MC2Rs are activated by ACTH binding and generate a cAMP response, the magnitude of which is dependent on the ambient ligand concentration. Expression of MRAP2 alongside MC2R promotes MC2R trafficking to the cell surface to a similar extent as MRAP; however, the resultant complex has an extremely low affinity for ACTH binding. As a result the response curve of cAMP generated plotted against ligand concentration is significantly shifted to the right when MC2R is co-expressed with MRAP2 compared to when MC2R is expressed with MRAP (Sebag and Hinkle, 2009a, 2010; Gorrigan et al., 2011). It was suggested that this difference was due to lack of the leucine, aspartic acid, tyrosine, isoleucine (LDYI) motif in MRAP2, which was present in MRAP, and insertion of the LDYI residues enabled the MRAP2/MC2R complex to respond to lower concentrations of ACTH (Sebag and Hinkle, 2009b). MRAP and MRAP2 are able to form heterodimeric complexes in vitro, however, whether such a complex is physiologically relevant is not known (Chan et al., 2009b; Sebag and Hinkle, 2010). In one study MC2R was expressed alongside both MRAP and MRAP2, the resulting dose response curve appeared sensitive to the ratio of MRAP to MRAP2, with increasing proportions of MRAP2 shifting the curve further to the right (Sebag and Hinkle, 2010). Importantly, this effect has not been observed in other studies (Chan et al., 2009b; Agulleiro et al., 2010). Interestingly, $\mathrm{Y} 1$ cells which are of murine adrenocortical origin and endogenously expresses MRAP, MRAP2 and MC2R generate cAMP in response to ACTH treatment and this effect can be abrogated by transient expression of MRAP2 within these cells (Sebag and Hinkle, 2010). Taken together, further work is required to determine if the expression levels of MRAP and MRAP2 in the adrenal gland could act as a physiological mechanism dictating ACTH responsiveness.

Mutations in MC2R and MRAP lead to the inherited condition FGD. In contrast, mutations in the MRAP2 gene have not been linked with this condition suggesting that it may not be involved in MC2R signaling in vivo and that any degree of functional redundancy between MRAP and MRAP2 is unable to completely compensate for MRAP dysfunction. In-keeping with this observation in situ hybridization studies and quantitative RT-PCR using rat tissue have demonstrated that MRAP is expressed at much higher levels than MRAP2 in adult adrenal glands (Gorrigan et al., 2011).

\section{MRAP2 BEYOND MC2R SIGNALING}

Consistent with the findings discussed above, exploring the interaction of MRAP with other members of the MCR family, MRAP2 has also been shown to interact with all five members of the MCR family by co-immunoprecipitation assays in transiently transfected cultured cells (Chan etal., 2009b). In one series of experiments when cell surface expression of individual MCRs was quantified in the presence or absence of MRAP2, expression levels of MC1R and MC3R were not affected by MRAP2 expression, however, expression levels of MC4R and MC5R were significantly reduced when they were co-expressed with MRAP2. Furthermore, when MCR signaling was examined in the presence of MRAP2, its expression was detrimental to MC3R, MC4R, and MC5R signaling but had no effect on MC1R signaling. In these experiments signaling was studied by quantifying cAMP generation in response to treatment with a single concentration of ligand. The effect of MRAP2 on MC4R signaling has been explored using a luciferase reporter assay to measure cAMP production across a range of ligand concentrations. In this study the authors concluded that although MC4R surface expression was reduced in the presence of MRAP2 that MC4R signaling did not appear to be affected (Sebag and Hinkle, 2010). The experiments discussed here have been conducted in $\mathrm{CHO}$ cells which do not endogenously express any of the components of the melanocortin system, further research will address whether MRAP2 interacts with MCRs beyond this experimental system and will further delineate the nature of these interactions. As the main site of MRAP2 expression is in brain and specifically in the hypothalamus it is likely that the main physiological function of MRAP2 is in the central nervous system, perhaps involving MC4R and/or MC3R.

\section{THE FUTURE OF MRAPS}

Numerous lines of genetic evidence suggest that subtle perturbations of signaling capacity at the level of MCRs can have pathological consequences. As our appreciation of the complex nature of the melanocortin system grows, a large family of molecules is emerging which impact upon signaling and confer the ability to integrate extracellular and intracellular cues into a co-ordinated biological signal. The discovery of two proteins which may interact with multiple members of the MCR family in distinct ways represents a significant advance in our understanding of melanocortin signaling and paves the way for further lines of research to explore the physiological roles of these proteins.

\section{ACKNOWLEDGMENTS}

Li F. Chan holds an MRC/Academy of Medical Sciences Clinician Scientist Fellowship [G0802796] and Rebecca J. Gorrigan is supported by a WellcomeTrust Research Training Fellowship [092024/Z/10/Z]. 


\section{REFERENCES}

Agulleiro, M. J., Roy, S., Sanchez, E., Puchol, S., Gallo-Payet, N., and Cerda-Reverter, J. M. (2010). Role of melanocortin receptor accessory proteins in the function of zebrafish melanocortin receptor type $2 . \mathrm{Mol}$. Cell. Endocrinol. 320, 145-152.

Balthasar, N., Dalgaard, L. T., Lee, C. E., Yu, J., Funahashi, H., Williams, T., etal. (2005). Divergence of melanocortin pathways in the control of food intake and energy expenditure. Cell 123, 493-505.

Beaumont, K. A., Smit, D. J., Liu, Y. Y., Chai, E., Patel, M. P., Millhauser, G. L., et al. (2012). Melanocortin-1 receptormediated signalling pathways activated by NDP-MSH and HBD3 ligands. Pigment Cell Melanoma Res. 25, 370-374.

Breit, A., Wolff, K., Kalwa, H., Jarry, H., Buch, T., and Gudermann, T. (2006). The natural inverse agonist agouti-related protein induces arrestin-mediated endocytosis of melanocortin-3 and -4 receptors. J. Biol. Chem. 281, 37447-37456.

Butler, A. A., Kesterson, R. A., Khong, K., Cullen, M. J., Pelleymounter, M. A., Dekoning, J., et al. (2000). A unique metabolic syndrome causes obesity in the melanocortin3 receptor-deficient mouse. Endocrinology 141, 3518-3521.

Chan, L. F., Metherell, L. A., Krude, H., Ball, C., O'Riordan, S. M. Costigan, C., et al. (2009a). Homozygous nonsense and frameshift mutations of the ACTH receptor in children with familial glucocorticoid deficiency (FGD) are not associated with long-term mineralocorticoid deficiency. Clin. Endocrinol. 71, 171-175.

Chan, L. F., Webb, T. R., Chung, T.T., Meimaridou, E., Cooray, S. N., Guasti, L., et al. (2009b). MRAP and MRAP2 are bidirectional regulators of the melanocortin receptor family. Proc. Natl. Acad. Sci. U.S.A. 106, 6146-6151.

Chen, A. S., Marsh, D. J., Trumbauer, M. E., Frazier, E. G., Guan, X. M., Yu, H., et al. (2000). Inactivation of the mouse melanocortin-3 receptor results in increased fat mass and reduced lean body mass. Nat. Genet. 26, 97-102.

Chen, W., Kelly, M. A., Opitz-Araya, X., Thomas, R. E., Low, M. J., and Cone, R. D. (1997). Exocrine gland dysfunction in MC5-R-deficient mice: evidence for coordinated regulation of exocrine gland function by melanocortin peptides. Cell 91 , 789-798.
Cheung, C. C., Clifton, D. K., and Steiner, R. A. (1997). Proopiomelanocortin neurons are direct targets for leptin in the hypothalamus. Endocrinology 138, 4489-4492.

Chung, T. T., Chan, L. F., Metherell, L. A., and Clark, A. J. (2010). Phenotypic characteristics of familial glucocorticoid deficiency (FGD) type 1 and 2. Clin. Endocrinol. 72 589-594.

Clark, A. J., Mcloughlin, L., and Grossman, A. (1993). Familial glucocorticoid deficiency associated with point mutation in the adrenocorticotropin receptor. Lancet 341, 461-462.

Cone, R. D. (2006). Studies on the physiological functions of the melanocortin system. Endocr. Rev. 27, 736-749.

Cooray, S. N., Almiro Do Vale, I., Leung, K.-Y., Webb, T. R., Chapple, J. P., Egertova, M., et al. (2008). The melanocortin 2 receptor accessory protein exists as a homodimer and is essential for the function of the melanocortin 2 receptor in the mouse Y1 cell line. Endocrinology 149 1935-1941.

Cooray, S. N., Chung, T. T., Mazhar, K., Szidonya, L., and Clark, A. J. (2011). Bioluminescence resonance energy transfer reveals the adrenocorticotropin (ACTH)-induced conformational change of the activated ACTH receptor complex in living cells. Endocrinology 152, 495-502.

Eipper, B. A., and Mains, R. E. (1980). Structure and biosynthesis of proadrenocorticotropin/endorphin and related peptides. Endocr. Rev. 1, 1-27.

Farooqi, I. S., Keogh, J. M., Yeo, G. S., Lank, E. J., Cheetham, T., and O’Rahilly, S. (2003). Clinical spectrum of obesity and mutations in the melanocortin 4 receptor gene. $N$. Engl. J. Med. 348, 1085-1095.

Gineau, L., Cognet, C., Kara, N., Lach, F. P., Dunne, J., Veturi, U., et al. (2012). Partial MCM4 deficiency in patients with growth retardation, adrenal insufficiency, and natural killer cell deficiency. J. Clin. Invest. 122, 821-832.

Gorrigan, R. J., Guasti, L., King, P., Clark, A. J. L., and Chan, L. F. (2011). Localisation of the melanocortin2-receptor and its accessory proteins in the developing and adult adrenal gland. J. Mol. Endocrinol. 46, 227-232.

Hoch, M., Eberle, A. N., Wagner, U., Bussmann, C., Peters, T., and Peterli, R. (2007). Expression and localization of melanocortin-1 receptor in human adipose tissues of severely obese patients. Obesity (Silver Spring) $15,40-49$.
Hoch, M., Hirzel, E., Lindinger, P. Eberle, A. N., Linscheid, P., Martin, I., et al. (2008). Weak functional coupling of the melanocortin-1 receptor expressed in human adipocytes. J. Recept. Signal Transduct. Res. 28, 485-504.

Hughes, C. R., Chung, T. T., Habeb, A. M., Kelestimur, F., Clark, A. J. and Metherell, L. A. (2010). Missense mutations in the melanocortin 2 receptor accessory protein that lead to late onset familial glucocorticoid deficiency type 2. J. Clin. Endocrinol. Metab. 95, 3497-3501.

Hughes, C. R., Guasti, L., Meimaridou, E., Chuang, C. H., Schimenti, J. C., King, P. J., et al. (2012). MCM4 mutation causes adrenal failure, short stature, and natural killer cell deficiency in humans. J. Clin. Invest. 122, 814-820.

Huszar, D., Lynch, C. A., FairchildHuntress, V., Dunmore, J. H. Fang, Q., Berkemeier, L. R., et al. (1997). Targeted disruption of the melanocortin-4 receptor results in obesity in mice. Cell 88, 131-141.

Jun, D. J., Na, K. Y., Kim, W., Kwak, D., Kwon, E. J., Yoon, J. H., et al. (2010). Melanocortins induce interleukin 6 gene expression and secretion through melanocortin receptors 2 and 5 in 3T3-L1 adipocytes. J. Mol. Endocrinol. 44, 225-236.

Lin, L., Hindmarsh, P. C., Metherell, L. A., Alzyoud, M., Al-Ali, M., Brain, C. E., et al. (2007). Severe loss-offunction mutations in the adrenocorticotropin receptor (ACTHR, MC2R) can be found in patients diagnosed with salt-losing adrenal hypoplasia. Clin. Endocrinol. 66, 205-210.

Lu, D., Willard, D., Patel, I. R., Kadwell, S., Overton, L., Kost, T., et al. (1994). Agouti protein is an antagonist of the melanocyte-stimulatinghormone receptor. Nature 371 , 799-802.

Meimaridou, E., Kowalczyk, J., Guasti, L., Hughes, C. R., Wagner, F., Frommolt, P., et al. (2012). Mutations in NNT encoding nicotinamide nucleotide transhydrogenase cause familial glucocorticoid deficiency. Nat. Genet. 44, 740-742.

Mencarelli, M., Dubern, B., Alili, R., Maestrini, S., Benajiba, L., Tagliaferri, M., et al. (2011). Rare melanocortin3 receptor mutations with in vitro functional consequences are associated with human obesity. Hum. Mol. Genet. 20, 392-399.

Metherell, L. A., Chapple, J. P., Cooray, S., David, A., Becker, C., Ruschendorf, F., et al. (2005). Mutations in MRAP, encoding a new interacting partner of the ACTH receptor, cause familial glucocorticoid deficiency type 2. Nat. Genet. 37, 166-170.

Metherell, L. A., Naville, D., Halaby, G., Begeot, M., Huebner, A., Nurnberg, G., et al. (2009). Nonclassic lipoid congenital adrenal hyperplasia masquerading as familial glucocorticoid deficiency. J. Clin. Endocrinol. Metab. 94, 3865-3871.

Moller, C. L., Raun, K., Jacobsen, M. L., Pedersen, T. A., Holst, B., Conde-Frieboes, K. W., et al. (2011). Characterization of murine melanocortin receptors mediating adipocyte lipolysis and examination of signalling pathways involved. Mol. Cell. Endocrinol. 341, 9-17.

Morgan, C., and Cone, R. D. (2006). Melanocortin-5 receptor deficiency in mice blocks a novel pathway influencing pheromone-induced aggression. Behav. Genet. 36, 291-300.

Morgan, C., Thomas, R. E., and Cone, R. D. (2004). Melanocortin-5 receptor deficiency promotes defensive behavior in male mice. Horm. Behav. 45, 58-63.

Mountjoy, K. G., Mortrud, M. T., Low, M. J., Simerly, R. B., and Cone, R. D. (1994). Localization of the melanocortin-4 receptor (MC4$\mathrm{R})$ in neuroendocrine and autonomic control circuits in the brain. Mol. Endocrinol. 8, 1298-1308.

Nijenhuis, W. A., Oosterom, J., and Adan, R. A. (2001). $\operatorname{AgRP}(83-$ 132) acts as an inverse agonist on the human-melanocortin-4 receptor. Mol. Endocrinol. 15, 164-171.

O’Riordan, S. M., Lynch, S. A., Hindmarsh, P. C., Chan, L. F., Clark, A. J., and Costigan, C. (2008). A novel variant of familial glucocorticoid deficiency prevalent among the Irish Traveler population. J. Clin. Endocrinol. Metab. 93, 2896-2899.

Patten, C. S., Daniels, D., Suzuki, A., Fluharty, S. J., and Yee, D. K. (2007). Structural and signaling requirements of the human melanocortin 4 receptor for MAP kinase activation. Regul. Pept. 142, 111-122.

Rodrigues, A. R., Pignatelli, D., Almeida, H., and Gouveia, A. M. (2009). Melanocortin 5 receptor activates ERK1/2 through a PI3K-regulated signaling mechanism. Mol. Cell. Endocrinol. 303, 74-81.

Roselli-Rehfuss, L., Mountjoy, K. G., Robbins, L. S., Mortrud, M. T., Low, M. J., Tatro, J. B., et al. (1993). Identification of a receptor for gamma melanotropin and other proopiomelanocortin peptides in the hypothalamus and limbic system. Proc. Natl. Acad. Sci. U.S.A. 90, 8856-8860.

Rumie, H., Metherell, L. A., Clark, A. J., Beauloye, V., and Maes, M. (2007). 
Clinical and biological phenotype of a patient with familial glucocorticoid deficiency type 2 caused by a mutation of melanocortin 2 receptor accessory protein. Eur. J. Endocrinol. 157, 539-542.

Schwartz, M. W., Seeley, R. J., Woods, S. C., Weigle, D. S., Campfield, L. A., Burn, P., etal. (1997). Leptin increases hypothalamic proopiomelanocortin mRNA expression in the rostral arcuate nucleus. Diabetes 46, 2119-2123.

Sebag, J. A., and Hinkle, P. M. (2007). Melanocortin-2 receptor accessory protein MRAP forms antiparallel homodimers. Proc. Natl. Acad. Sci. U.S.A. 104, 20244-20249.

Sebag, J. A., and Hinkle, P. M. (2009a). Opposite effects of the melanocortin2 (MC2) receptor accessory protein MRAP on MC2 and MC5 receptor dimerization and trafficking. J. Biol. Chem. 284, 22641-22648.

Sebag, J. A., and Hinkle, P. M. (2009b). Regions of melanocortin 2 (MC2) receptor accessory protein necessary for dual topology and MC2 receptor trafficking and signaling. J. Biol. Chem. 284, 610-618.

Sebag, J. A., and Hinkle, P. M. (2010). Regulation of $\mathrm{G}$ proteincoupled receptor signaling: specific dominant-negative effects of melanocortin 2 receptor accessory protein 2. Sci. Signal. 3, ra28.

Seng Lee, Y., Kok Seng Poh, L., Lay Kee Kek, B., and Yin Loke, K. (2007). The role of melanocortin 3 receptor gene in childhood obesity. Diabetes $56,2622-2630$.
Sutton, G. M., Begriche, K., Kumar, K. G., Gimble, J. M., Perez-Tilve, D., Nogueiras, R., et al. (2010). Central nervous system melanocortin-3 receptors are required for synchronizing metabolism during entrainment to restricted feeding during the light cycle. FASEB J. 24, 862-872.

Sutton, G. M., Duos, B., Patterson, L. M., and Berthoud, H. R. (2005). Melanocortinergic modulation of cholecystokinin-induced suppression of feeding through extracellular signal-regulated kinase signaling in rat solitary nucleus. Endocrinology 146, 3739-3747.

Sutton, G. M., Perez-Tilve, D. Nogueiras, R., Fang, J., Kim, J. K., Cone, R. D., et al. (2008). The melanocortin-3 receptor is required for entrainment to meal intake. $J$. Neurosci. 28, 12946-12955.

Swope, V. B., Jameson, J. A., Mcfarland, K. L., Supp, D. M., Miller W. E., Mcgraw, D. W., et al. (2012). Defining MC1R regulation in human melanocytes by its agonist alphamelanocortin and antagonists agouti signaling protein and beta-defensin 3 . J. Invest. Dermatol. 132, 2255-2262.

Tsigos, C., Arai, K., Hung, W., and Chrousos, G. P. (1993). Hereditary isolated glucocorticoid deficiency is associated with abnormalities of the adrenocorticotropin receptor gene. $J$. Clin. Invest. 92, 2458-2461.

Turan, S., Hughes, C., Atay, Z., Guran, T., Haliloglu, B., Clark, A. J., et al. (2012). An atypical case of familial glucocorticoid deficiency without pigmentation caused by coexistent homozygous mutations in MC2R (T152K) and MC1R (R160W). J. Clin. Endocrinol. Metab. 97, E771E774.

Vage, D. I., Lu, D., Klungland, H., Lien, S., Adalsteinsson, S., and Cone, R. D. (1997). A non-epistatic interaction of agouti and extension in the fox, Vulpes vulpes. Nat. Genet. 15, 311-315.

Vaisse, C., Clement, K., Guy-Grand, B. and Froguel, P. (1998). A frameshift mutation in human MC4R is associated with a dominant form of obesity. Nat. Genet. 20, 113-114.

Valverde, P., Healy, E., Jackson, I., Rees, J. L., and Thody, A. J. (1995). Variants of the melanocyte-stimulating hormone receptor gene are associated with red hair and fair skin in humans. Nat. Genet. 11, 328-330.

Vongs, A., Lynn, N. M., and Rosenblum, C. I. (2004). Activation of MAP kinase by MC4-R through PI3 kinase. Regul. Pept. 120, 113-118.

Webb, T. R., Chan, L., Cooray, S. N., Cheetham, M. E., Chapple, J. P., and Clark, A. J. L. (2009). Distinct melanocortin 2 receptor accessory protein domains are required for melanocortin 2 receptor interaction and promotion of receptor trafficking. Endocrinology 150, 720-726.

Willer, C. J., Speliotes, E. K., Loos, R. J., Li, S., Lindgren, C. M., Heid, I. M., et al. (2009). Six new loci associated with body mass index highlight a neuronal influence on body weight regulation. Nat. Genet. 41, 25-34.

$\mathrm{Xu}$, A., Choi, K. L., Wang, Y., Permana, P. A., Xu, L. Y., Bogardus,
C., etal. (2002). Identification of novel putative membrane proteins selectively expressed during adipose conversion of 3T3-L1 cells. Biochem. Biophys. Res. Commun. 293, 11611167.

Yeo, G. S., Farooqi, I. S., Aminian, S., Halsall, D. J., Stanhope, R. G., and O'Rahilly, S. (1998). A frameshift mutation in MC4R associated with dominantly inherited human obesity. Nat. Genet. 20, 111-112.

Conflict of Interest Statement: The authors declare that the research was conducted in the absence of any commercial or financial relationships that could be construed as a potential conflict of interest.

Received: 02 November 2012; accepted: 25 January 2013; published online: 08 February 2013.

Citation: Ramachandrappa S, Gorrigan RJ, Clark AJL and Chan LF (2013) The melanocortin receptors and their accessory proteins. Front. Endocrin. 4:9. doi: 10.3389/fendo.2013.00009

This article was submitted to Frontiers in Neuroendocrine Science, a specialty of Frontiers in Endocrinology.

Copyright (C) 2013 Ramachandrappa, Gorrigan, Clark and Chan. This is an open-access article distributed under the terms of the Creative Commons Attribution License, which permits use, distribution and reproduction in other forums, provided the original authors and source are credited and subject to any copyright notices concerning any thirdparty graphics etc. 